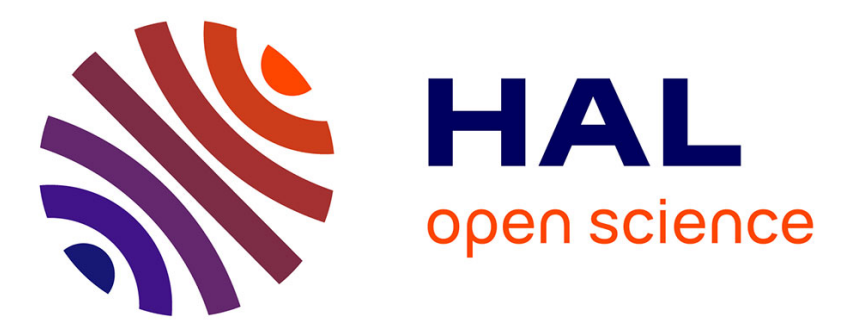

\title{
An Extension of Unstructured-PEEC Method to Magnetic Media
}

R. Torchio, F. Moro, Gérard Meunier, Jean-Michel Guichon, Olivier Chadebec

\section{To cite this version:}

R. Torchio, F. Moro, Gérard Meunier, Jean-Michel Guichon, Olivier Chadebec. An Extension of Unstructured-PEEC Method to Magnetic Media. IEEE Transactions on Magnetics, 2019, 55 (6), 10.1109/TMAG.2018.2889435 . hal-02350715

\section{HAL Id: hal-02350715 https://hal.science/hal-02350715}

Submitted on 6 Nov 2019

HAL is a multi-disciplinary open access archive for the deposit and dissemination of scientific research documents, whether they are published or not. The documents may come from teaching and research institutions in France or abroad, or from public or private research centers.
L'archive ouverte pluridisciplinaire HAL, est destinée au dépôt et à la diffusion de documents scientifiques de niveau recherche, publiés ou non, émanant des établissements d'enseignement et de recherche français ou étrangers, des laboratoires publics ou privés. 


\title{
An Extension of Unstructured-PEEC Method to Magnetic Media
}

\author{
R. Torchio ${ }^{1,2}$, F. Moro ${ }^{1}$, G. Meunier ${ }^{2}$, J.-M. Guichon ${ }^{2}$, and O. Chadebec ${ }^{2}$ \\ ${ }^{1}$ Università degli Studi di Padova, Dipartimento di Ingegneria Industriale, Padova, Italy \\ ${ }^{2}$ Univ. Grenoble Alpes, CNRS, Grenoble INP, G2Elab, 38000 Grenoble, France
}

\begin{abstract}
An extension of the unstructured Partial Element Equivalent Circuit (PEEC) method to magnetic media is proposed. Linear inhomogeneous conductive, dielectric, and magnetic media are considered. By formulating PEEC in terms of div-free currents, an efficient circuit algorithm based on the independent loop search or basis reduction scheme can be used to solve the electromagnetic problem. In such a way, a limited number of unknowns can be used and non-simply connected domains can be treated as well. The formulation is validated on a real $\mathrm{L}-\mathrm{C}-\mathrm{T}$ component against measurements and numerical results from commercial software based on Integral Equation Method and Finite Element Method.
\end{abstract}

Index Terms-PEEC, integral equations, EMC, magnetic media, L-C-T component.

\section{INTRODUCTION}

The Partial Element Equivalent Circuit (PEEC) method is a well-known Integral Equation Method (IEM) suitable for solving electromagnetic (EM) problems coupled to external electric circuits. The PEEC method has been first introduced by A. Ruehli in 1972 for orthogonal grids and conductive media. In literature two different approaches can be found: the Standard-PEEC [1] and the Unstructured-PEEC (or Dual$P E E C)$ [2]. In the standard approach, regular hexahedral and quadrilateral elements are usually adopted for the mesh discretization [1]. The current density and the charge density distributions are expanded by means of pulse basis functions and the Electric Field Integral Equation (EFIE), combined with Ohm's law and the continuity equation, is then discretized by using a Galerkin approach. In [3] and [4] the Standard-PEEC has then been extended to nonorthogonal mesh and dielectric and magnetic media.

The Unstructured-PEEC method was first introduced in [2] for surface conductive models. This approach provides an exact and accurate formalization of the PEEC tessellation, allowing the study of structured and unstructured mesh without any particular additional effort. With the Unstructured-PEEC method the properties of the EM unknowns are naturally and strongly imposed. Recently, the method has been extended to volume media and dielectric materials [5]. The extension of Unstructured-PEEC to magnetic media was first proposed in [6] for low frequency cases and in [7] for the whole frequency range by introducing the magnetization and the amperian currents as unknowns. However, this approach is limited to the study of homogeneous media and simply connected regions; moreover, it requires a great amount of unknowns when magnetic media are considered.

This work proposes an extension to magnetic media of the formulation previously presented in [5]. The unknowns are div-free total electric and magnetic currents expanded by means of Whitney face elements. The proposed formulation allows for homogeneous and inhomogeneous media as well. The div-free condition of the current vectors can be strongly

Manuscript received December 1, 2012; revised August 26, 2015. Corresponding author: R. Torchio (email: riccardo.torchio@studenti.unipd.it). imposed by using an independent loop search algorithm [8] or a basis reduction scheme [9], which also reduces the number of unknowns. Moreover, both simply and non-simply connected regions can be considered. The formulation is here developed considering the time delay effects. The formulation is validated on the case of the $\mathrm{L}-\mathrm{C}-\mathrm{T}$ (integrated inductor $(\mathrm{L})$, capacitor (C), and transformer (T)) component presented in [10] against measurements, FEM and IEM software.

\section{FORMULATION}

Conductive, dielectric, and magnetic domains are first defined as $\Omega_{c}, \Omega_{d}$, and $\Omega_{m}$, respectively. As it is customary, the terms dielectric and magnetic indicate media with permittivity and permeability different from those of the vacuum, respectively. The union between conductive and dielectric domains $\Omega_{e}=\Omega_{c} \cup \Omega_{d}$ is defined as the electric domain. The disjoint union between the electric and the magnetic domains defines the interior region $\Omega=\Omega_{e} \sqcup \Omega_{m}$, (computational domain). The boundaries of these domains are: $\Gamma_{c}=\partial \Omega_{c}, \Gamma_{d}=\partial \Omega_{d}$, $\Gamma_{m}=\partial \Omega_{m}, \Gamma_{e}=\partial \Omega_{e}$, and $\Gamma=\partial \Omega$.

Following [11], when conductive, dielectric, and magnetic media are considered, full Maxwell's equations can be written in the following form, avoiding the use of the electric displacement $\mathbf{D}$ and the magnetic flux density $\mathbf{B}$, i.e.

$$
\begin{aligned}
\nabla \cdot \mathbf{E} & =\varepsilon_{0}^{-1} \varrho_{e}, & -\nabla \times \mathbf{E} & =\mathbf{J}_{m}+i \omega \mu_{0} \mathbf{H}, \\
\nabla \cdot \mathbf{H} & =\mu_{0}^{-1} \varrho_{m}, & \nabla \times \mathbf{H} & =\mathbf{J}_{e}+i \omega \varepsilon_{0} \mathbf{E},
\end{aligned}
$$

where $\mathbf{E}$ is the electric field, $\mathbf{H}$ is the magnetic field, $\mathbf{J}_{e}$ is the electric current density $\left(\mathbf{J}_{e}=\mathbf{J}_{c}+\mathbf{J}_{p}\right.$, where $\mathbf{J}_{c}$ is the conduction current density and $\mathbf{J}_{p}=i \omega \mathbf{P}$ is the polarization current density), $\mathbf{J}_{m}=i \omega \mu_{0} \mathbf{M}$ is the magnetization current density [11]. $\varrho_{e}$ is the electric charge density $\left(\varrho_{e}=\varrho_{c}+\right.$ $\varrho_{p}$, where $\varrho_{c}$ is the free-charge density and $\varrho_{p}$ is the bound polarization charge density), $\varrho_{m}$ is the bound magnetization charge density, $\omega$ is the angular frequency, $\varepsilon_{0}$ and $\mu_{0}$ are the permittivity and permeability of vacuum, respectively. $\mathbf{P}$ and $\mathbf{M}$ are the polarization and magnetization vectors, respectively. Maxwell's equations (1) are complemented by the following constitutive relationships [11]: 


$$
\begin{gathered}
\mathbf{E}=\frac{\mathbf{J}_{c}}{\sigma_{c}} \text { in } \Omega_{c}, \quad \mathbf{E}=\frac{\mathbf{J}_{p}}{i \omega \varepsilon_{0}\left(\varepsilon_{r}-1\right)} \text { in } \Omega_{d}, \\
\mathbf{H}=\frac{\mathbf{J}_{m}}{i \omega \mu_{0}\left(\mu_{r}-1\right)} \text { in } \Omega_{m},
\end{gathered}
$$

where $\sigma_{c}$ is the electric conductivity, $\varepsilon_{r}$ is the relative electric permittivity, $\mu_{r}$ is the relative magnetic permeability, and $x$ is the position point (the field point). According to [12], the electric and magnetic fields satisfying (1) can be written as

$$
\begin{aligned}
\mathbf{E} & =-i \omega \mathbf{A}_{e}-\nabla \varphi_{e}-\varepsilon_{0}^{-1} \nabla \times \mathbf{A}_{m}+\mathbf{E}_{0}, \\
\mathbf{H} & =-i \omega \mathbf{A}_{m}-\nabla \varphi_{m}+\mu_{0}^{-1} \nabla \times \mathbf{A}_{e}+\mathbf{H}_{0},
\end{aligned}
$$

where $\mathbf{A}_{e}$ and $\mathbf{A}_{m}$ are the electric and magnetic Hertz vector potentials scaled by $j \omega$, respectively, whereas $\varphi_{e}$ and $\varphi_{m}$ are the electric and magnetic Hertz scalar potentials, respectively. $\mathbf{E}_{0}$ and $\mathbf{H}_{0}$ are the external electric and magnetic fields produced by some source domain $\Omega_{0}$, exterior to $\Omega$.

After imposing Lorenz gauge as $\nabla \cdot \mathbf{A}_{e}=-i \omega \varepsilon_{0} \mu_{0} \varphi_{e}, \nabla$. $\mathbf{A}_{m}=-i \omega \varepsilon_{0} \mu_{0} \varphi_{m}$, and combining (1) and (3), the following differential equations are obtained:

$$
\begin{gathered}
\nabla^{2} \mathbf{A}_{e}-\left(i \omega c_{0}^{-1}\right)^{2} \mathbf{A}_{e}=-\mu_{0} \mathbf{J}_{e}, \\
\nabla^{2} \mathbf{A}_{m}-\left(i \omega c_{0}^{-1}\right)^{2} \mathbf{A}_{m}=-\varepsilon_{0} \mathbf{J}_{m},
\end{gathered}
$$

where $c_{0}$ is the speed of light in vacuum. The solutions of equations (4) are given by the following integral expressions

$$
\begin{aligned}
\mathbf{A}_{e}(x) & =\mu_{0} \int_{\Omega} \mathbf{J}_{e}(y) g(x, y) d v_{y}, \\
\mathbf{A}_{m}(x) & =\varepsilon_{0} \int_{\Omega} \mathbf{J}_{m}(y) g(x, y) d v_{y},
\end{aligned}
$$

where $g(x, y)=e^{-i \omega\|x-y\| / c_{0}} /\|x-y\|$ is the retarded Green function, $x$ is the field point, and $y$ is the integration point.

From (5), applying the Lorenz gauge condition and Green's identities, the integral expressions of the scalar electric and magnetic Hertz potentials are obtained:

$$
\begin{gathered}
\varphi_{e}(x)=\left(i \omega \varepsilon_{0}\right)^{-1} \int_{\Omega} \mathbf{J}_{e}(y) \cdot \nabla^{\prime} g(x, y) d v_{y}, \\
\varphi_{m}(x)=\left(i \omega \mu_{0}\right)^{-1} \int_{\Omega} \mathbf{J}_{m}(y) \cdot \nabla^{\prime} g(x, y) d v_{y},
\end{gathered}
$$

where the prime symbol denotes gradient with respect to $y$. Inserting equations (2), (5), and (6) in (3), the following integral equations are obtained:

$$
\begin{aligned}
\mathbf{E}_{0}(x) & =\frac{\mathbf{J}_{e}(x)}{\sigma_{e}(x)}+i \omega \mu_{0} \int_{\Omega} \mathbf{J}_{e}(y) g(x, y) d v_{y} \\
& +\left(i \omega \varepsilon_{0}\right)^{-1} \nabla \int_{\Omega} \mathbf{J}_{e}(y) \cdot \nabla^{\prime} g(x, y) d v_{y} \\
& +\varepsilon_{0}^{-1} \nabla \times\left(\varepsilon_{0} \int_{\Omega} \mathbf{J}_{m}(y) g(x, y) d v_{y}\right) i n \Omega_{e}, \\
\mathbf{H}_{0}(x) & =\frac{\mathbf{J}_{m}(x)}{\sigma_{m}(x)}+i \omega \varepsilon_{0} \int_{\Omega} \mathbf{J}_{m}(y) g(x, y) d v_{y} \\
& +\left(i \omega \mu_{0}\right)^{-1} \nabla \int_{\Omega} \mathbf{J}_{m}(y) \cdot \nabla^{\prime} g(x, y) d v_{y} \\
& -\mu_{0}^{-1} \nabla \times\left(\mu_{0} \int_{\Omega} \mathbf{J}_{e}(y) g(x, y) d v_{y}\right) i n \Omega_{m},
\end{aligned}
$$

where $\sigma_{e}=\sigma_{c}$ in $\Omega_{c}, \sigma_{e}=i \omega \varepsilon_{0}\left(\varepsilon_{r}-1\right)$ in $\Omega_{d}$, and $\sigma_{m}=i \omega \mu_{0}\left(\mu_{r}-1\right)$ in $\Omega_{m}$. In the same fashion of the formulation previously developed in [5], div-free total electric and magnetic currents are introduced as problem unknowns:

$$
\mathbf{J}_{e}^{t o t}=\mathbf{J}_{e}+i \omega \varepsilon_{0} \mathbf{E}, \quad \mathbf{J}_{m}^{t o t}=\mathbf{J}_{m}+i \omega \mu_{0} \mathbf{H} .
$$

By combining (2) and (9) the following relationships hold:

$$
\mathbf{J}_{e}=\frac{\sigma_{e}}{\sigma_{e}^{*}} \mathbf{J}_{e}^{t o t} \text { in } \Omega_{e}, \quad \mathbf{J}_{m}=\frac{\sigma_{m}}{\sigma_{m}^{*}} \mathbf{J}_{m}^{t o t} \text { in } \Omega_{m},
$$

where $\sigma_{e}^{*}=\sigma_{c}+i \omega \varepsilon_{0} \varepsilon_{r}$ is the equivalent electric conductivity and $\sigma_{m}^{*}=i \omega \mu_{0} \mu_{r}$ is the equivalent magnetic conductivity.

Final electric and magnetic integral equations are obtained by inserting (10) in (7) and (8). The discretization of these equations is discussed below.

\section{DisCRETIZATION}

First, the computational domain $\Omega$ is discretized into tetrahedral or hexahedral elements. The total electric and magnetic current densities are expanded by means of face elements, as:

$$
\mathbf{J}_{e}^{t o t}(x)=\sum_{k e=1}^{N_{f e}} \mathbf{w}_{k e}(x) j_{e_{k e}}^{t o t}, \mathbf{J}_{m}^{t o t}(x)=\sum_{k m=1}^{N_{f m}} \mathbf{w}_{k m}(x) j_{m_{k m}}^{t o t},
$$

where $N_{f e}$ and $N_{f m}$ are the number of faces of $\Omega_{e}$ and $\Omega_{m}$, respectively, $\mathbf{w}_{k e}$ and $\mathbf{w}_{k m}$ are the Whitney 2 -form functions [13] related to $f_{k e}$ and $f_{k m}$ (the $k e-$ th and $k m$-th faces of the mesh of $\Omega_{e}$ and $\Omega_{m}$, respectively), $j_{e_{k e}}^{t o t}$ is the the flux of $\mathbf{J}_{e}^{t o t}$ through $f_{k e}$, and $j_{m_{k m}}^{t o t}$ is the flux of $\mathbf{J}_{m}^{t o t}$ through $f_{k m} . j_{e_{k e}}^{t o t}$ and $j_{m_{k m}}^{\text {tot }}$ are coefficients of the arrays $\mathbf{j}_{e}^{\text {tot }}$ and $\mathbf{j}_{m}^{\text {tot }}$, respectively. Whitney elements can be defined on tetrahedra [13] or 8-nodes hexahedra (with planar quadrilateral faces) [14]. Following [5], the arrays of degrees of freedom (DoFs) $\phi_{e}=\left[\phi_{e}^{v} ; \phi_{e}^{s}\right]$ related to the averaged electric scalar potential, are defined as:

$$
\phi_{e_{k e}}^{v}=\int_{v_{k e}} \frac{\varphi_{e}(x)}{V_{k e}} d v_{x}, \quad \phi_{e_{k e}}^{s}=\int_{f_{k e}} \frac{\varphi_{e}(x)}{S_{k e}} d s_{x},
$$

where $V_{k e}$ is the volume of the $k e-$ th tetrahedral or hexahedral element $v_{k e}$, and $S_{k e}$ is the area of the boundary face $f_{k e}$; indeed, $\phi_{e}^{s}$ is only defined for the faces that discretize $\Gamma_{e}$. Equivalent definitions can be applied to the averaged magnetic scalar potential, $\phi_{m}=\left[\boldsymbol{\phi}_{m}^{v} ; \boldsymbol{\phi}_{m}^{s}\right]$.

Testing (7) and (8) with the face Whitney elements and using (10) and (11) results in:

$$
\begin{aligned}
& {\left[\mathbf{R}_{e}+i \omega \mathbf{L}_{e}\right] \mathbf{j}_{e}^{t o t}+\mathbf{G}_{e} \phi_{e}+\mathbf{K}_{e m} \mathbf{j}_{m}^{t o t}=\mathbf{e}_{0},} \\
& {\left[\mathbf{R}_{m}+i \omega \mathbf{L}_{m}\right] \mathbf{j}_{m}^{t o t}+\mathbf{G}_{m} \boldsymbol{\phi}_{m}-\mathbf{K}_{m e} \mathbf{j}_{e}^{t o t}=\mathbf{h}_{0},}
\end{aligned}
$$

where $\mathbf{R}_{e}, \mathbf{L}_{e}$, and $\mathbf{G}_{e}$ are the electric resistance, inductance, and incidence matrices, respectively, whereas $\mathbf{R}_{m}, \mathbf{L}_{m}$, and $\mathbf{G}_{m}$ are the corresponding magnetic ones. $\mathbf{e}_{0}$ and $\mathbf{h}_{0}$ are the DoFs related to $\mathbf{E}_{0}$ and $\mathbf{H}_{0}: e_{0_{h e}}=\int_{\Omega_{e}} \mathbf{w}_{h e}(x) \cdot \mathbf{E}_{0}(x) d v_{x}$ and $h_{0_{h m}}=\int_{\Omega_{m}} \mathbf{w}_{h m}(x) \cdot \mathbf{H}_{0}(x) d v_{x}$.

Likewise [5], the discrete form of (6) is given by $i \omega \phi_{e}=$ $\mathbf{P}_{e} \mathbf{j}_{e}^{t o t}$ and $i \omega \phi_{m}=\mathbf{P}_{m} \mathbf{j}_{m}^{t o t}$, where $\mathbf{P}_{e}$ is the electric potential matrix and $\mathbf{P}_{m}$ the magnetic one. Matrix $\mathbf{P}_{e}$ can be partitioned as $\mathbf{P}_{e}=\left[\mathbf{P}_{e}^{v} ; \mathbf{P}_{e}^{s}\right]$, where $\mathbf{P}_{e}^{v}$ and $\mathbf{P}_{e}^{s}$ are the volume and surface potential matrices, respectively. Thus, $i \omega \phi_{e}^{v}=\mathbf{P}_{e}^{v} \mathbf{j}_{e}^{t o t}$ 
way. The integral expressions of the matrix coefficients are:

$$
\begin{aligned}
R_{e_{h e, k e}} & =\int_{\Omega_{e}} \frac{\mathbf{w}_{h e}(x) \cdot \mathbf{w}_{k e}(x)}{\sigma_{e}^{*}(y)} d v_{y} d v_{x}, \\
L_{e_{h e}, k e} & =\mu_{0} \int_{\Omega_{e}} \int_{\Omega_{e}} \frac{\sigma_{e}(y)}{\sigma_{e}^{*}(y)} \mathbf{w}_{h e}(x) \cdot \mathbf{w}_{k e}(y) g(x, y) d v_{y} d v_{x}, \\
P_{e_{h e, k e}}^{v} & =\frac{1}{\varepsilon_{0} V_{v_{h e}}} \int_{v_{h e}} \int_{\Omega_{e}} \frac{\sigma_{e}(y)}{\sigma_{e}^{*}(y)} \mathbf{w}_{k e}(y) \cdot \nabla^{\prime} g(x, y) d v_{y} d v_{x}, \\
P_{e_{h e, k e}}^{s} & =\frac{1}{\varepsilon_{0} S_{f_{h e}}} \int_{f_{h e}} \int_{\Omega_{e}} \frac{\sigma_{e}(y)}{\sigma_{e}^{*}(y)} \mathbf{w}_{k e}(y) \cdot \nabla^{\prime} g(x, y) d v_{y} d s_{x} .
\end{aligned}
$$

Completely equivalent expressions can be obtained for the magnetic matrices $\mathbf{R}_{m}, \mathbf{L}_{m}, \mathbf{P}_{m}^{v}$, and $\mathbf{P}_{m}^{s} . \mathbf{K}_{e m}$ and $\mathbf{K}_{m e}$ are the matrices representing the EM coupling between electric and magnetic media. The coefficients of these matrices can be obtained by applying the curl operator to (5):

$$
\begin{aligned}
& K_{e m_{h e, k m}}=\frac{1}{4 \pi} \int_{\Omega_{e}} \int_{\Omega_{m}} \mathbf{w}_{h e}(x) \cdot \frac{\sigma_{m}(y)}{\sigma_{m}^{*}(y)} \\
& \left(\frac{\mathbf{w}_{k m}(y) \times(x-y)}{\|x-y\|^{3}}+i \omega \frac{\mathbf{w}_{k m}(y) \times(x-y)}{c_{0}\|x-y\|^{2}}\right) d v_{y} d v_{x},
\end{aligned}
$$

and similarly for $\mathbf{K}_{m e}$. The inductance and potential coefficients in (14) can be evaluated numerically when the two supports of the integrals do not overlap, (far-mutual coefficients). When the two supports overlap (self and nearmutual coefficients) the evaluation of a double integral with singular integrand function is required. Therefore, Singularity Extraction Techniques (SET) must be applied in order to evaluate analytically the inner integral and numerically the outer integral [15]. For electrically small objects the time delay effects are negligible, thus $g(x, y) \approx \frac{1}{\|x-y\|}$. This approximation simplifies the evaluation of (14) and a computationally less expensive SET can be adopted. Moreover, the second term inside the inner integral of (15) can be neglected.

Matrix $\mathbf{G}_{e}$ and $\mathbf{G}_{m}$ are incidence matrices representing the algebraic equivalent of the gradient operator. $\mathbf{G}_{e}$ is $N_{f e} \times\left(N_{v e}+N_{f_{b} e}\right)$, where $N_{f e}$ and $N_{f_{b} e}$ are the number of the faces and boundary faces of the mesh of $\Omega_{e}$, respectively. Each row of $\mathbf{G}_{e}$ related to an internal face, $f_{e}^{\text {int }}$, has +1 and -1 in the columns corresponding to the two tetrahedral (or hexahedral) elements sharing the face $f_{e}^{\text {int }}$. Instead, each row of $\mathbf{G}_{e}$ related to an external face, $f_{e}^{\text {ext }}$, has +1 in the column corresponding to the boundary face $f_{e}^{e x t}$, and -1 in the column corresponding to the tetrahedral/hexahedral element having the face $f_{e}^{e x t}$. Similar considerations apply to $\mathbf{G}_{m}$.

Finally, (13) can be written as

$$
\left[\begin{array}{cc}
\mathbf{Z}_{e} & \mathbf{K}_{e m} \\
-\mathbf{K}_{m e} & \mathbf{Z}_{m}
\end{array}\right]\left[\begin{array}{c}
\mathbf{j}_{e}^{t o t} \\
\mathbf{j}_{m}^{t o t}
\end{array}\right]=\left[\begin{array}{c}
\mathbf{e}_{0} \\
\mathbf{h}_{0}
\end{array}\right]
$$

where $\mathbf{Z}_{e}=\mathbf{R}_{e}+i \omega \mathbf{L}_{e}+(i \omega)^{-1} \mathbf{G}_{e} \mathbf{P}_{e}$ and $\mathbf{Z}_{m}=\mathbf{R}_{m}+$ $i \omega \mathbf{L}_{m}+(i \omega)^{-1} \mathbf{G}_{m} \mathbf{P}_{m}$.

In order to strongly impose the div-free condition on $\mathbf{J}_{e}^{t o t}$ and $\mathbf{J}_{m}^{t o t}$, i.e. the Kirchhoff's Current Law on the unknowns of (16), a set of independent currents is found. This problem can be addressed by interpreting (13) as the coupling of two circuit matrix equations (an electric one and a magnetic one).
The equivalent electric/magnetic circuit is built as follows: any face of the mesh is interpreted as an electric/magnetic circuit branch, each volume and boundary face is considered as an electric/magnetic circuit node. Thus, (16) can be solved by using a circuital approach based on the independent loop search [8] or basis reduction scheme [9]:

$$
\mathbf{Q}^{T}\left[\begin{array}{cc}
\mathbf{Z}_{e} & \mathbf{K}_{e m} \\
-\mathbf{K}_{m e} & \mathbf{Z}_{m}
\end{array}\right] \mathbf{Q}\left[\begin{array}{c}
\mathbf{j}_{e}^{o} \\
\mathbf{j}_{m}^{o}
\end{array}\right]=\mathbf{Q}^{T}\left[\begin{array}{c}
\mathbf{e}_{0} \\
\mathbf{h}_{0}
\end{array}\right],
$$

where $\mathbf{Q}=\operatorname{diag}\left(\mathbf{Q}_{e}, \mathbf{Q}_{m}\right), \mathbf{j}_{e}^{t o t}=\mathbf{Q}_{e} \mathbf{j}_{e}^{o}$, and $\mathbf{j}_{m}^{t o t}=\mathbf{Q}_{m} \mathbf{j}_{m}^{o}$, in which $\mathbf{Q}_{e}$ and $\mathbf{Q}_{m}$ (obtained as explained in [8] or [9]) are the change of basis matrices and $\mathbf{j}_{e}^{o}$ and $\mathbf{j}_{m}^{o}$ are the independent currents. Unlike [7], where the use of discrete potentials and tree-gauging requires special techniques when non-simply connected domains are considered, the approach here proposed is based on a circuital interpretation of (13), which naturally handles non-simply connected domains.

Thanks to the circuit interpretation, the proposed PEEC formulation can be easily coupled with external circuits. Thus, a Kirchhoff's voltage law written for each external circuit element must be solved together with (13). Then, the independent loop search algorithm for the construction of $\mathbf{Q}_{e}$ operates on the augmented circuit graph made by the union of the equivalent electric circuit and the external circuit elements.

\section{NuMERICAL RESULtS}

The Unstructured-PEEC code has been developed with MATLAB $\cap$ and parallel MEX-FORTRAN functions based on OpenMP libraries. The simulations have been run on a Windows machine equipped with 6-core/24-thread processors (Intel Xeon E5645 @2.40GHz).

The $\mathrm{L}-\mathrm{C}-\mathrm{T}$ component proposed in [10] is here considered. All geometrical and material details of the device are reported in [16] (Appendix 4). The device is made by 16 copper layers $(70 \mu \mathrm{m}$ thickness each) that compose the first and the second part of the primary winding of the transformer. 16 copper layers are printed on dielectric substrates made
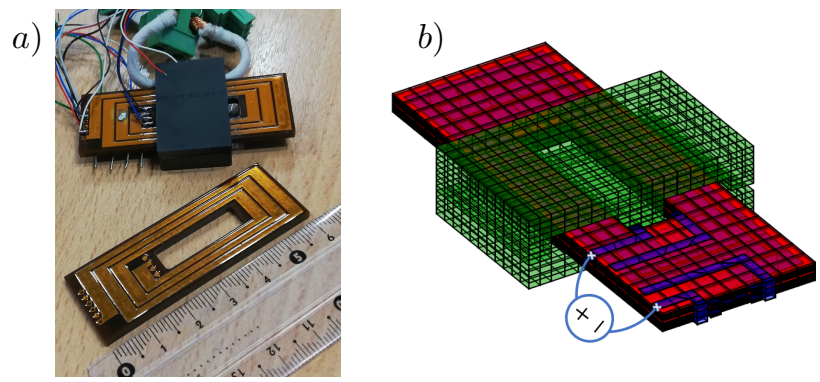

Fig. 1: a) L-C-T prototype. b) L-C-T Model.

by Kapton $\left(\varepsilon_{r}=3.3,50 \mu \mathrm{m}\right.$ thick $)$ or Preg $\left(\varepsilon_{r}=4.5\right.$, $140 \mu \mathrm{m}$ thick). The magnetic core is made by Ferrite $3 \mathrm{~F} 3$. Fig. 1 shows a picture of the $\mathrm{L}-\mathrm{C}-\mathrm{T}$ component and its corresponding model. Note that neither the dielectric nor the magnetic media are simply connected regions. The device is fed by a voltage source connected to only two of the four terminals of the primary winding, (Fig. 1). At low frequency, the device behaves as a capacitor. The primary winding is an 
open circuit and the current flows from the first part to the second part of the winding thanks to capacitive effects [10]. In Fig. 2, the magnitude and the argument of the equivalent

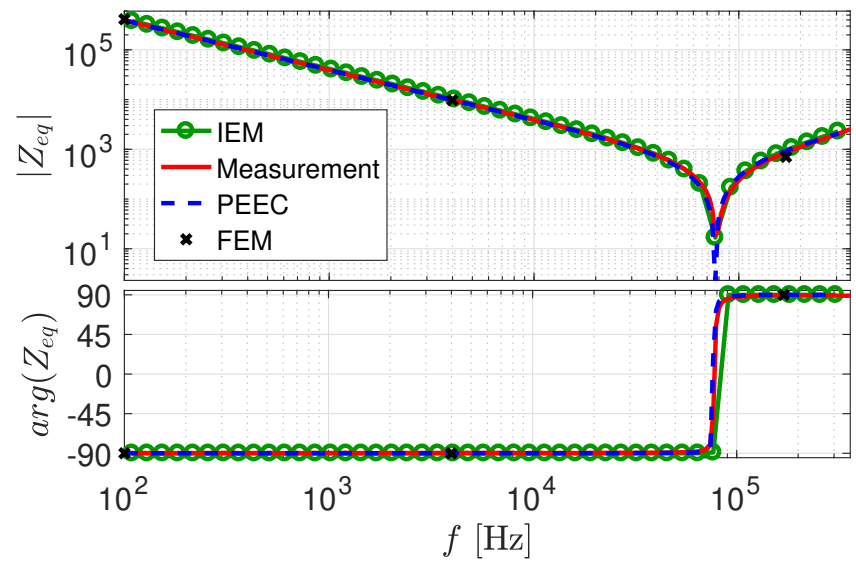

Fig. 2: $Z_{e q}$ magnitude and phase of the L-C-T component.

impedance $\left(Z_{e q}\right)$ obtained from the PEEC code are compared with measurements and with the numerical results obtained from FEM and IEM commercial software.

Fig. 3 shows the cases of the device without the the magnetic core (no-core) and the case with the conductive media only (con-only).

The PEEC model of the (entire) L-C-T component is made by hexahedral elements and consists of 18,429 DoFs. The time required for the computation of the matrices is $545 \mathrm{~s}$. The matrix system is solved by means of LU decomposition and the solution time for a single frequency value is $733 \mathrm{~s}$ with a Peak Memory Usage (PMU) of 15 GB. For a frequency sweep

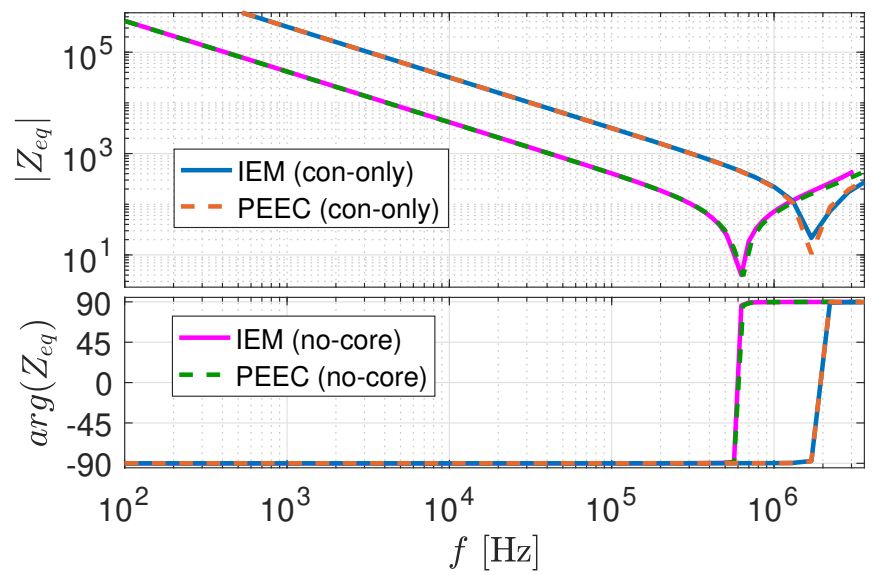

Fig. 3: $Z_{e q}$ magnitude and phase, no-core and con-only cases.

analysis, the PEEC matrices can be computed only once. Then, for each frequency value, the system (17) is assembled and solved.

In order to assess the computational cost required by FEM for the study of the L-C-T component, three simulations have been run. Due to the small thickness of the layers a great amount of elements is needed for the discretization. The FEM problem is first solved by adopting liner basis functions $(4,956,667$ DoFs) and using a direct solver (MUMPS). This required 8,914 $\mathrm{s}$ and a PMU of $380 \mathrm{~GB}$. Then, starting from the obtained solution, an iterative solver (FGMRES) and quadratic basis functions are adopted to increase the solution accuracy (29,582,652 DoFs, 9,799 s, PMU of 430 GB).

The model adopted for the commercial IEM software consists of 35,243 DoFs and the mesh is made by tetrahedral and triangular elements. An equivalent surface model is used for the copper layers to include in the model the voltage excitation. The simulations requires $1,892 \mathrm{~s}$ and a PMU of 19 GB for each frequency value.

\section{CONCLUSION}

An extension to magnetic media of the Unstructured-PEEC method, first introduced in [5], has been presented. Unlike [7], the proposed formulation allows for non-simply connected domains and a smaller number of unknowns is required when magnetic media are involved. Moreover, thanks to the use of div-free total currents, inhomogeneous media can be considered without any additional computational effort. The proposed method has been successfully tested by simulating an L-C-T component. Further researches concerning the use of compression techniques combined with preconditioning and iterative methods are under developments to alleviate the memory requirements.

\section{REFERENCES}

[1] A. E. Ruehli et al., Circuit Oriented Electromagnetic Modeling Using the PEEC Techniques. John Wiley \& Sons, Inc., June 2017.

[2] F. Freschi and M. Repetto, "A general framework for mixed structured/unstructured PEEC modelling," Applied Computational Electromagnetic Society Journal, ACES, vol. 23, no. 3, pp. 200-206, 2008.

[3] A. E. Ruehli et al., "Nonorthogonal PEEC formulation for time- and frequency-domain EM and circuit modeling," IEEE Trans. Electromagn. Compat., vol. 45, no. 2, pp. 167-176, May 2003.

[4] D. Romano and G. Antonini, "Quasi-static partial element equivalent circuit models of magneto-dielectric materials", IET Microwaves, Antennas \& Propagation, vol. 11, no. 6, pp. 915-922, 2017.

[5] J. Siau et al., "Volume integral formulation using face elements for electromagnetic problem considering conductors and dielectrics," IEEE Trans. on Electromag. Compat., vol. 58, no. 5, pp. 1587-1594, Oct. 2016.

[6] G. Meunier et al., "A Magnetic Flux-Electric Current Volume Integral Formulation Based on Facet Elements for Solving Electromagnetic Problems," IEEE Trans. on Magn., vol. 51, no. 3, Mar. 2015.

[7] R. Torchio et al., "A 3-D PEEC formulation based on the cell method for full wave analyses with conductive, dielectric, and magnetic media," IEEE Trans. Magn., 2017.

[8] T.-S. Nguyen et al., "An independent loops search algorithm for solving inductive PEEC large problems," Progr. Electromagn. Res., vol. 23, pp. 53-63, Jan. 2012.

[9] M.-K. Li and W. C. Chew, "Applying divergence-free condition in solving the volume integral equation," Progr. Electromagn. Res., vol. 57, pp. 311-333, 2006.

[10] Y. Lembeye et al., "Integrated planar L-C-T component: Design,Characterization and Experimental Efficiency analysis," IEEE Trans. Power Electron., vol. 20, no. 3, pp. 593-599, May 2005.

[11] R. M. Fano et al., Electromagnetic Fields, Energy, and Forces. M.I.T. Press, 1960.

[12] A. Nisbet, "Herzian electromagnetic potentials and associated gauge transformation," Proc. Math. Phys. Eng. Sci., 1955.

[13] A. Bossavit, Computational Electromagnetism. Academic Press, 1998.

[14] P. Dular et al., "Constitutive matrices using hexahedra in a discrete approach for eddy currents," 2006 12th Biennial IEEE Conference on Electromagnetic Field Computation, Miami, FL, 2006, pp. 185-185.

[15] S. Järbenpää et al., "Singularity extraction technique for integral equation methods with larger order basis functions on plane triangles and tetrahedra," Int. J. Numer. Meth. Eng., vol. 58, pp. 1149-1165, 2003.

[16] P. Goubier, Etude et Conception de composants passifs $L-C-T$ intégrés. Ph.D. dissertation, Univ. of Grenoble, Grenoble, France, 2003. 